\title{
¿Qué influye en el uso de Apps? Un estudio en el contexto de la pandemia COVID-19, en Loja-Ecuador
}

\section{What influences the use of Apps? A study in the context of the COVID-19 pandemic, in Loja Ecuador}

\author{
Viviana Chamba-González 1 https://orcid.org/0000-0002-5381-2032, \\ Diana Encalada ${ }^{1}$ https://orcid.org/0000-0001-6462-1277, Milenny Soto-Alvarado 1 https://orcid.org/0000- \\ 0003-2259-0091, María del Cisne Tituaña-Castillo 1 https://orcid.org/0000-0003-3043-8436
}

${ }^{1}$ Departamento de Economía, Universidad Técnica Particular de Loja, Loja, Ecuador lvchamba4@utpl.edu.ec, dencaladaleutpl.edu.ec,

mjsoto@utpl.edu.ec, mdtituania@utpl.edu.ec

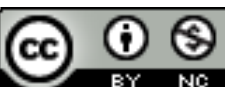

Esta obra está bajo una licencia internacional Creative Commons Atribución-NoComercial 4.0. $\begin{array}{ll}\text { Enviado: } & 2021 / 11 / 09 \\ \text { Aceptado: } & 2021 / 12 / 22 \\ \text { Publicado: } & 2021 / 12 / 30\end{array}$

\section{Resumen}

La digitalización de la economía ha tomado un rol preponderante en el desarrollo económico, acentuado por la pandemia COVID-19 al restringir las interacciones físicas de la población. En este contexto, las aplicaciones móviles se han identificado como un modo de acceso dominante para la conectividad individual, transformando el estilo de vida de la población y el funcionamiento de la economía. Este estudio explora el uso de las aplicaciones móviles en Loja - Ecuador durante el último año y examina los factores que influyeron en su uso. Esto se logró aplicando una encuesta estructurada en línea y un modelo econométrico de respuesta ordinal. Los principales hallazgos sugieren que aspectos sociodemográficos, como la generación a la que pertenecen y el nivel de educación, y factores relacionados con el uso y descarga de aplicaciones (Apps), como el uso de Facebook y Zoom, influyen significativamente en el uso de aplicaciones móviles. El estudio presenta, además, información que pueda orientar un mejor uso de las aplicaciones móviles en el marco de la economía creativa.

Palabras clave: aplicación de telecomunicaciones, economía cultural, economía de la ciencia, pandemia.

Sumario: Introducción, Metodología, Resultados y Discusión y Conclusiones.

Como citar: Chamba-González, V., Encalada, D., Soto-Alvarado, M., \& Tituaña-Castillo, M. (2021). ¿Qué influye en el uso de Apps? Un estudio en el contexto de la pandemia COVID-19, en Loja-Ecuador. Revista

Tecnológica - Espol, 33(3), 56-67. http://www.rte.espol.edu.ec/index.php/tecnologica/article/view/882 


\begin{abstract}
The digitalization of the economy has taken a leading role in economic development, accentuated by the COVID-19 pandemic by restricting the physical interactions of the population. In this context, mobile applications have been identified as a dominant mode of access for individual connectivity, transforming the lifestyle of the population and the functioning of the economy. This study explores the use of mobile applications in Loja Ecuador, during the last year and examines the factors that influenced their use. This was achieved by applying a structured online survey and an ordinal response econometric model. The main findings suggest that sociodemographic aspects, such as their generation and level of education, and factors related to the use and downloading of applications (Apps), such as the use of Facebook and Zoom, significantly influence the use of mobile applications. The study also presents information that can guide a better use of mobile applications within the framework of the creative economy.
\end{abstract}

Keywords: telecommunications application, cultural economy, science economy, pandemic.

\title{
Introducción
}

La sociedad moderna se ha caracterizado por un rápido desarrollo de las tecnologías de la información y comunicación (TICs), las que se han convertido en una parte integral de la estructura de la sociedad al virtualizar las comunicaciones sociales y el acceso a los recursos. Hoy en día, el uso de TICs es un requisito previo para establecer cualquier tipo de comunicación a nivel global (Horoshko et al., 2021). En 2019, el 41\% de la población mundial adquirió teléfonos inteligentes (Statista, 2020c) y en 2020 el recuento de descargas de aplicaciones móviles había superado los 218 mil millones (Statista, 2020a). Al mismo tiempo, en 2020, en la tienda de Google Play estuvieron disponibles 2,56 millones de aplicaciones móviles, mientras que, en la tienda de Apple estuvieron disponibles 1,85 millones de aplicaciones móviles (Statista, 2020b). En lo que respecta a las plataformas sociales, las más utilizadas en el mundo son Facebook, YouTube y WhatsApp. El 35\% de los usuarios de estas plataformas las utiliza para estar al día con eventos actuales y el 37\% para estar informados (We Are Social Inc \& Hootsuite, 2021).

En medio de esta revolución tecnológica e interacción humano-tecnología, surge la digitalización de la economía, cuyas raíces se remontan a alrededor de la década de 1990 con la aparición del Internet, que transformó la estructura económica e industrial (Schwab, 2017). Después de la llegada de la revolución industrial, la digitalización ha tomado un papel protagónico en el desarrollo económico de los países, aumentando su productividad, promoviendo el desarrollo industrial y generando importantes cambios sociales. Esta transformación sociocultural dio lugar a la economía digital, definida por Kumar \& Kumar Yadav (2015) como la red internacional de actividades sociales y económicas facilitadas por las tecnologías digitales como la red móvil y de Internet.

La economía digital representa la fusión de varias iniciativas de propósito general, con acciones sociales y económicas de amplio espectro e iniciativas relacionadas con el uso de tecnología. Este componente de la economía integra los sistemas físicos (enrutadores, líneas y banda ancha); los dispositivos empleados para accesibilidad (teléfonos inteligentes y computadoras); aplicaciones que alimentan softwares (Salesforce y Google) y las funcionalidades que pueden proporcionar a los usuarios (tecnología de la información, computación en la nube y análisis de datos) (Báez \& Yariv Brauner, 2018).

De acuerdo con el Banco Interamericano de Desarrollo (BID), la economía digital constituye tanto un desafío estratégico como una oportunidad única para mejorar la calidad de 
vida de las personas en los países en desarrollo a través de una mejora en los servicios públicos y consecuentemente un aumento en la productividad (BID, 2019). Esta economía engloba tres componentes principales: infraestructura de apoyo, procesos comerciales electrónicos (nuevas formas de hacer negocios) y transacciones comerciales (compra y venta de bienes y servicios en línea) (Mesenbourg, 2001).

Dentro del conjunto de nuevas tecnologías de rápida difusión se cuenta con: i) los dispositivos de usuario final como los teléfonos inteligentes, tabletas y netbooks; ii) modelos comerciales como la computación en la nube y plataformas digitales; y, iii) los datos digitales, las tecnologías de automatización y la robótica (Li et al., 2020). Las Apps, que corresponden al segundo grupo, son programas de software que funcionan en teléfonos inteligentes, tabletas y computadoras, y cuyo acceso se vincula esencialmente a su descarga a través de las tiendas virtuales como App Store (iOS) o Google Play (Android). En general, este tipo de software es habitualmente generado por desarrolladores de tecnologías móviles para diferentes usos segmentados, vinculados con el entretenimiento, la educación, la información, entre otros (Santamaría-Puerto y Hernández-Rincón, 2015).

Las Apps, también identificadas como una parte del ecosistema de la economía digital, facilitan el comercio electrónico, el acceso a servicios de comunicación y tienen un amplio uso, particularmente en dispositivos móviles, los que se han convertido en un modo de acceso dominante para la conectividad individual, transformando, en los últimos años, el estilo de vida y el comercio digital (Fallis, 2013). En el 2020, a nivel mundial, las descargas de aplicaciones móviles aumentaron en un 7\%; siendo TikTok la de mayor descarga, seguida de Facebook, WhatsApp, Zoom Cloud Meeting e Instagram. En el mundo, las aplicaciones con mayor número de usuarios son Facebook (96\%), YouTube (56\%) e Instagram (38\%) (We Are Social Inc \& Hootsuite, 2021).

En los últimos años el uso de aplicaciones móviles se ha incrementado a una velocidad sin precedentes (Celleri \& Garay, 2021). En el 2020, según el informe "Digital 2021", cerca del $54 \%$ de la población mundial fue usuario activo en redes sociales y el $99 \%$ de estos accedieron a través de sus teléfonos celulares (We Are Social Inc \& Hootsuite, 2021). El Instituto Vodafone para la Sociedad y las Comunicaciones (2013) reportó que el incremento al acceso de telefonía móvil estaba directamente relacionado con un mayor uso de aplicaciones móviles, las que han tenido un mayor impacto desde el lanzamiento de la App Store de Apple en 2008 y, debido a que Google y Microsoft también establecieron sus propios mercados de aplicaciones. Actualmente, Play Store superó a Apple Store, al registrar 50 mil millones de descargas en comparación con las 48 mil millones que registró Apple.

Países como Reino Unido se han convertido en una "sociedad de teléfonos inteligentes". En 2019 el 93\% de los británicos de 18 a 44 años tenía un teléfono inteligente (Deloitte, 2019) y 7 de cada 10 usaban Apps descargadas en un dispositivo móvil, diseñadas para realizar un propósito específico (OFCOM, 2016). Este gran impacto que han tenido las Apps en la sociedad ha motivado la investigación sobre diferentes temas relacionados con su uso en diferentes sectores. Por ejemplo, Aydin et al (2015) encontró que los usuarios de atención médica utilizan cada vez más aplicaciones con este propósito. Rahimi et al., (2021) determinó que el género, la edad, el nivel de educación, la actitud frente a la tecnología y el miedo a contraer COVID-19 influyeron en la intención de usar este tipo de Apps. Con respecto a aplicaciones de actividad física, el uso es mayor en mujeres entre 25 y 50 años que residen en zonas donde la mayor parte de la población tiene un nivel socioeconómico bajo (Pontin et al., 2021). 
En el sector educativo, Morales et al. (2020) concluyeron que, en Colombia, existe una mayor tendencia a que las mujeres jóvenes de las carreras de ingenierías utilicen Apps móviles en las aulas de clase, y que variables como la edad y la carrera no influyen en su uso. En el sector financiero, donde también se ha generalizado el uso de Apps para optimizar el tiempo que los clientes emplean en trasladarse a sus oficinas corporativas y usar personalmente los servicios que ofrecen, un estudio, basado en el modelo de aceptación de tecnología (TAM por sus siglas en inglés), de amplio respaldo empírico (Chuttur, 2009; Kabbiri et al., 2018; Luarn \& Lin, 2005; Rezaei et al., 2020), determinó que los factores que influyen en el uso de aplicaciones móviles de las instituciones financieras son la facilidad de uso percibida, utilidad percibida, compatibilidad del estilo de vida, innovación personal hacia las tecnologías de la información y el interés de aceptación y uso de nuevas tecnologías (Bernal Peralta et al., 2018).

Li et al., (2006), basado en la premisa de que el consumidor maximiza su utilidad sujeta no sólo a restricciones de ingresos sino también a restricciones de tiempo, encontró que la comodidad que ofrecen el internet y las Apps es un determinante de las compras en línea. Explica que las compras en tiendas de Internet van un paso más allá de las físicas al eliminar casi por completo costos de tiempo. El tiempo gastado es el dedicado a navegar por los sitios web.

La pandemia COVID-19, originada en China a principios de diciembre de 2019, se ha convertido en una de las enfermedades más infecciosas en la historia de la humanidad (OMS, 2020), con gran impacto en la vida cotidiana de las personas en todo el mundo, acelerando la adopción y uso de herramientas digitales en línea. Su masiva propagación y rigurosas medidas de prevención han acelerado el crecimiento de las economías digitales a nivel mundial, y es probable que su efecto vaya más allá del tiempo que esta dure (Li et al., 2020).

De acuerdo con la Unión Internacional de Telecomunicaciones (ITU, por sus siglas en inglés), la crisis de la COVID-19 ha puesto de relieve el papel fundamental que desempeñan las tecnologías de la información y la comunicación en el funcionamiento continuo de las sociedades (ITU, 2021). De hecho, en la mayoría de los países se implementaron Apps de salud para monitorear los síntomas y rastrear los posibles casos de COVID-19.

De otro lado, es necesario ampliar el análisis de los factores que determinan el uso de las Apps a un nivel generacional. El término "generación" se emplea para definir al "grupo de edad que comparte a lo largo de su historia un conjunto de experiencias formativas que lo distinguen de sus predecesores" (Bonvalet \& Ogg, 2006). Analizar los fenómenos generacionales permite comprender la influencia de los eventos históricos en el comportamiento y convicción compartida de un grupo poblacional (Lombardía et al., 2008). La clasificación y las principales características de las generaciones, según el Pew Research Center (2019), se resumen en la Tabla 1.

El desarrollo de investigaciones relacionadas con los determinantes del uso de aplicaciones móviles es escaso y particularmente limitado en el contexto de la pandemia COVID-19. La mayoría de los estudios se han enfocado en análisis nacionales y sectoriales; por tanto, el presente estudio busca contribuir con un análisis generalizado y localizado del uso de Apps en el marco de la COVID-19. De manera específica, el objetivo es determinar y analizar los factores que influyen en el uso de aplicaciones móviles durante la pandemia COVID-19, utilizando un modelo de respuesta ordinal. Se espera que los resultados encontrados contribuyan al diseño de políticas públicas que optimicen el uso de las Apps y mejoren el bienestar social de la población. 
Tabla 1

Clasificación de las generaciones

\begin{tabular}{|c|c|c|c|}
\hline GENERACIÓN & $\begin{array}{c}\text { EDAD } \\
\text { EN } 2021\end{array}$ & HITOS DESTACADOS & CARACTERÍSTICAS \\
\hline $\begin{array}{l}\text { Generación } \\
\text { Silenciosa }\end{array}$ & $\begin{array}{l}76 \text { a } 95 \\
\text { años }\end{array}$ & $\begin{array}{l}\text { La Gran Depresión } \\
\text { Segunda Guerra Mundial }\end{array}$ & $\begin{array}{l}\text { - } \quad \text { Son conservadores y tradicionalistas. } \\
\text { - } \quad \text { Prefieren trabajar de forma individual y se caracterizan por } \\
\text { su dedicación y lealtad a sus actividades laborales. } \\
\text { - } \quad \text { Su ideal es construir una vida en familia. }\end{array}$ \\
\hline Baby Boomers & $\begin{array}{l}57 \text { a } 75 \\
\text { años }\end{array}$ & $\begin{array}{l}\text { Finalización de la Segunda } \\
\text { Guerra Mundial } \\
\text { Baby Boom (época de } \\
\text { marcado incremento en el } \\
\text { nacimiento de niños) }\end{array}$ & 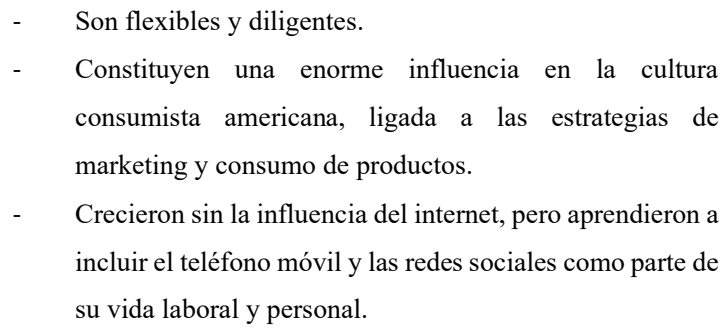 \\
\hline Generación X & $\begin{array}{l}41 \text { a } 56 \\
\text { años }\end{array}$ & $\begin{array}{l}\text { Revolución informática } \\
\text { (Descubrimiento del } \\
\text { Internet) }\end{array}$ & $\begin{array}{l}\text { - Crecieron en un hogar en donde los padres trabajaban o } \\
\text { estaban divorciados. } \\
\text { - } \quad \text { Su entorno estaba marcado por inseguridades familiares. } \\
\text { - } \quad \text { Tienden a ser sobreprotectores con sus hijos con el fin de } \\
\text { garantizarles estabilidad familiar. }\end{array}$ \\
\hline $\begin{array}{l}\text { Generación Y } \\
\text { (Millenials) }\end{array}$ & $\begin{array}{l}25 \text { a } 40 \\
\text { años }\end{array}$ & $\begin{array}{l}\text { "Lunes negro" (colapso de la } \\
\text { Bolsa de New York) } \\
\text { El colapso de la Unión } \\
\text { Soviética y con ello, el final } \\
\text { de la Guerra Fría. }\end{array}$ & $\begin{array}{l}\text { - Se caracterizan por el uso de la tecnología como parte } \\
\text { integral de su estilo de vida, pues, crecieron inmersos en } \\
\text { el internet, los teléfonos inteligentes y las redes sociales. } \\
\text { Es la primera generación que entra al mercado laboral con } \\
\text { mayor y mejor conocimiento de las herramientas de } \\
\text { negocios que sus superiores. } \\
\text { Quieren cambiar el mundo, ser más correctos, más } \\
\text { honestos, más ecológicos, más orgánicos, más exitosos, } \\
\text { son ciudadanos de un mundo globalizado. } \\
\text { Es la generación cuyas características son más similares } \\
\text { entre países que las de cualquier otra generación. }\end{array}$ \\
\hline $\begin{array}{l}\text { Generación Z } \\
\text { (Centennials) }\end{array}$ & $\begin{array}{l}9 \text { a } 24 \\
\text { años }\end{array}$ & $\begin{array}{ll}\text { - } & \text { Ataque terrorista del } 11 \text { de } \\
\text { septiembre } \\
\text { - } & \text { Nacimiento de la red social } \\
\text { Facebook y crecimiento } \\
\text { acelerado de Google } \\
\text { - } \quad \text { Colapso de la burbuja } \\
\text { inmobiliaria en Estados } \\
\text { Unidos }\end{array}$ & $\begin{array}{l}\text { - Son considerados "nativos digitales", nacidos en un } \\
\text { mundo de alta tecnología y con el servicio de internet al } \\
\text { alcance de la mano. } \\
\text { - Los teléfonos inteligentes ocupan un papel central en su } \\
\text { vida (pueden llegar a pasar un promedio de } 4 \text { horas y } 15 \\
\text { minutos por día en su teléfono móvil) } \\
\text { - Son independientes y tienen preferencia para emprender. } \\
\text { - } \quad \text { Es una "generación activista", pues centran su atención en } \\
\text { resolver los problemas sociales y políticos mundiales } \\
\text { enfocados especialmente en la sostenibilidad, medio } \\
\text { ambiente y maltrato animal. }\end{array}$ \\
\hline
\end{tabular}

Nota. Adaptado de Díaz-Sarmiento et al., (2017), New Strategist Publication (2010), Saucedo Soto et al., (2017), Stein (2013), PWC (2011), Wey-Smola y Sutton (2002).

En los siguientes apartados del estudio se exponen la metodología, los resultados, análisis y discusión de resultados y las conclusiones, limitaciones y perspectivas de futuras investigaciones. 


\section{Entorno del estudio y participantes}

\section{Metodología}

El estudio se desarrolló en Loja, una ciudad con 251951 habitantes (INEC, 2021), ubicada al sur de Ecuador. Utilizando el método de muestreo intencional, y la fórmula de la muestra para poblaciones finitas, se entrevistaron en línea a 386 usuarios de Apps móviles.

\section{Recopilación de datos}

Los datos fueron obtenidos mediante una encuesta estructurada, diseñada en base a la encuesta de CRITEO (2020). El cuestionario constaba de 3 secciones. La primera sección recopiló datos sobre la descarga y uso de Apps, la segunda sección investigaba sobre permisos de privacidad y confianza en la seguridad de las Apps, y la tercera sección recogía las características sociodemográficas de los encuestados. El cuestionario incluyó preguntas abiertas y cerradas y se emplearon escalas de Likert en varias preguntas. Previa su aplicación, la encuesta fue piloteada y validada. En abril de 2021 se aplicó la encuesta utilizando el programa ArcGis Survey 123. La información obtenida fue sistematizada y analizada en los programas Excel y Stata versión 15. Las variables utilizadas en el análisis se describen en la Tabla 2.

\section{Tabla 2}

Descripción de las variables

\begin{tabular}{|c|c|c|c|c|c|c|}
\hline VARIABLE & TIPO & VALORES & MEDIA & $\begin{array}{c}\text { DESV. } \\
\text { EST. }\end{array}$ & MIN & MAX \\
\hline Número de Apps descargadas & Discreta & & 1.264 & 2.491 & 0 & 20 \\
\hline \multirow{4}{*}{ Uso de las Apps } & \multirow{4}{*}{ Ordinal } & $0=$ poco & & & & \\
\hline & & $1=$ algo & & & & \\
\hline & & $2=$ bastante & & & & \\
\hline & & $3=$ mucho & & & & \\
\hline \multirow{2}{*}{ Aumento del uso de redes sociales } & \multirow{2}{*}{ Nominal } & $1=\mathrm{si}$ & & & & \\
\hline & & $0=$ no & & & & \\
\hline \multirow{2}{*}{ Uso de Facebook } & \multirow{2}{*}{ Nominal } & $1=\mathrm{si}$ & & & & \\
\hline & & $0=$ no & & & & \\
\hline \multirow{2}{*}{ Uso de Zoom } & \multirow{2}{*}{ Nominal } & $1=\mathrm{si}$ & & & & \\
\hline & & $0=$ no & & & & \\
\hline \multirow{2}{*}{ Atención a los anuncios de las Apps } & \multirow{2}{*}{ Nominal } & $1=\mathrm{si}$ & & & & \\
\hline & & $0=$ no & & & & \\
\hline \multirow{2}{*}{ Género } & \multirow{2}{*}{ Nominal } & $1=$ hombre & & & & \\
\hline & & $0=$ mujer & & & & \\
\hline Edad & Continua & & 31 & 12.41 & 15 & 77 \\
\hline \multirow{5}{*}{ Generaciones } & \multirow{5}{*}{ Ordinal } & 1=generación $\mathrm{Z}$ & & & & \\
\hline & & $2=$ generación $Y$ & & & & \\
\hline & & $3=$ generación $X$ & & & & \\
\hline & & 4=baby boom y & & & & \\
\hline & & silenciosa & & & & \\
\hline \multirow{2}{*}{ Nivel de educación } & \multirow{2}{*}{ Ordinal } & 1=básica & & & & \\
\hline & & $2=$ primaria & & & & \\
\hline
\end{tabular}




\begin{tabular}{|c|c|c|c|c|c|c|}
\hline VARIABLE & TIPO & VALORES & MEDIA & $\begin{array}{c}\text { DESV. } \\
\text { EST. }\end{array}$ & MIN & MAX \\
\hline & & $3=$ secundaria & & & & \\
\hline & & 4=técnica & & & & \\
\hline & & $5=$ superior & & & & \\
\hline \multirow{5}{*}{ Estado civil } & \multirow{5}{*}{ Nominal } & $1=$ soltero & & & & \\
\hline & & $2=$ casado & & & & \\
\hline & & 3=unión libre & & & & \\
\hline & & $4=$ divorciado & & & & \\
\hline & & $5=$ viudo & & & & \\
\hline \multirow{2}{*}{ Estado civil - soltero } & \multirow{2}{*}{ Nominal } & $1=\mathrm{si}$ & & & & \\
\hline & & $0=$ no & & & & \\
\hline \multirow{7}{*}{ Ocupación } & \multirow{7}{*}{ Nominal } & $1=$ empleado privado & & & & \\
\hline & & 2=empleado público & & & & \\
\hline & & $3=$ por cuenta propia & & & & \\
\hline & & 4=ama de casa & & & & \\
\hline & & $5=$ estudiante & & & & \\
\hline & & $6=$ jubilado & & & & \\
\hline & & $7=$ desempleado & & & & \\
\hline \multirow{2}{*}{ Ocupación - estudiante } & \multirow{2}{*}{ Nominal } & $1=\mathrm{si}$ & & & & \\
\hline & & $0=$ no & & & & \\
\hline \multirow{8}{*}{ Ingresos } & \multirow{8}{*}{ Ordinal } & $1=0$ & & & & \\
\hline & & $2=1-400$ & & & & \\
\hline & & $3=401-1000$ & & & & \\
\hline & & $4=1001-2000$ & & & & \\
\hline & & $5=2001-3000$ & & & & \\
\hline & & $6=3001-4000$ & & & & \\
\hline & & $7=4001-5000$ & & & & \\
\hline & & $8=\geq 5000$ & & & & \\
\hline
\end{tabular}

\section{Análisis de datos}

La regresión logística es uno de los enfoques estadísticos más comunes para modelar una variable de resultado categórica en dependencia de un conjunto de variables predictoras. Este modelo amplía un modelo de regresión logística para tener en cuenta múltiples variables dependientes de clasificación (Tutz and Berger, 2017). El modelo es adecuado para los casos en los que la variable dependiente es ordinal e incluye al menos 3 categorías ordenadas (Fuks and Salazar, 2008). Específicamente, el modelo estima las probabilidades de que una persona utilice aplicaciones móviles. La variable de resultado "uso de Apps móviles" es ordinal y tiene 4 categorías. Las variables, dependiente e independientes, se muestran en la Tabla 2.

\section{Resultados y Discusión}

Los 386 encuestados incluyen más mujeres (55\%) que hombres (45\%). La edad media fue de 31 años. La mayor parte presentó un nivel de educación medio $(20 \%)$ o superior $(63 \%)$ y estaban solteros (61\%). Un poco más de la tercera parte de los encuestados $(35 \%)$ tenían un empleo, el $14 \%$ trabajaban por cuenta propia y el $40 \%$ tenían como ocupación principal estudiar. El ingreso promedio mensual del $29 \%$ de los encuestados estaba entre \$ 401 y $\$ 1000$, 
para el $27 \%$ fue igual o menor al salario básico unificado ecuatoriano (\$400) y un poco más de la cuarta parte de los encuestados fueron dependientes económicamente (25.6\%).

Tomando 11 de las variables descritas en la Tabla 2 como variables independientes $<$ y el uso de las Apps como variable dependiente, se estimaron una serie de modelos de regresión logística ordenados. La mejor estimación obtenida se muestra en la Tabla 3. Los estadísticos de chi-cuadrado se utilizaron para verificar el modelo y los estadísticos de chi-cuadrado de Wald se utilizaron para verificar la significancia de la variable, cuya influencia es estadísticamente significativa $(\mathrm{p}>0.5)$.

Tabla 3

Resultados del análisis de regresión logística ordenada

\begin{tabular}{lccc}
\hline \multicolumn{1}{c}{ VARIABLE } & COEFICIENTE & ERROR ESTÁNDAR & VALOR - P \\
\hline Género & 0.300 & 0.206 & 0.144 \\
Generaciones & $-0.712^{* * *}$ & 0.202 & 0.000 \\
Estado civil - soltero & -0.149 & 0.285 & 0.602 \\
Nivel de educación & $0.233^{*}$ & 0.107 & 0.029 \\
Ingreso & 0.057 & 0.037 & 0.130 \\
Ocupación-estudiante & -0.504 & 0.285 & 0.077 \\
Número de Apps descargadas & $0.106^{* *}$ & 0.038 & 0.005 \\
Aumento del uso de redes sociales & 0.454 & 0.325 & 0.162 \\
Uso de Facebook & $1.002^{* *}$ & 0.398 & 0.012 \\
Uso de Zoom & $0.544^{*}$ & 0.276 & 0.049 \\
Atención a los anuncios de las Apps & -0.144 & 0.094 & 0.126 \\
\hline$*$ p $<0.05, * * \mathrm{p}<0.01, * * * \mathrm{p}<0.001$ & & &
\end{tabular}

Las variables generaciones, nivel de educación, número de Apps descargadas, uso de Facebook y uso de Zoom presentaron importancia estadística en el uso de las Apps móviles durante el primer año de la COVID-19. La variable generaciones fue estadísticamente significativa a un nivel de confianza del $99,9 \%$ y presentó signo negativo, es decir, mientras más antigua sea la generación a la que pertenece el usuario, menos probabilidad tiene de aumentar el uso de Apps móviles. Los coeficientes de las variables número de Apps descargadas, y uso de Facebook fueron significativos a un nivel de confianza del 99\% y presentaron signo positivo, lo que indica que, mientras más Apps descargadas durante la pandemia tengan los usuarios en sus dispositivos móviles y sean usuarios de la red social Facebook, mayor será la probabilidad de incrementar el uso de Apps móviles. En cuanto al nivel de educación y uso de Zoom, estas variables presentaron coeficientes con un nivel de confianza del $95 \%$ y signo positivo, lo que sugiere que mientras mayor educación tengan los usuarios y utilicen Zoom, mayor probabilidad tendrán de aumentar el uso de Apps móviles.

Es importante mencionar que variables como el ingreso presentaron un signo positivo esperado y significancia a un nivel de confianza del $90 \%$, es decir, mientras mayor ingreso perciben los usuarios, mayor probabilidad tendrán de aumentar el uso de las Apps móviles. Otras variables como el género y el aumento del uso de redes sociales durante el primer año de pandemia, cuyo coeficiente no es significativo, presentaron signo positivo, lo que sugiere que los hombres tienen una mayor probabilidad de uso con respecto a las mujeres, así como quienes han aumentado el uso de redes sociales de quienes no lo han hecho. Con respecto a las variables estado civil - soltero y atención a los anuncios de las Apps, también con coeficientes no significativos, presentaron un signo negativo, lo que indica que los usuarios solteros y que 
prestan atención a los anuncios de las Apps, tienen una menor probabilidad de aumentar el uso de Apps móviles respecto a los de otro estado civil y a quienes no prestan atención a los anuncios de éstas.

En cuanto a la relación entre la variable dependiente con las independientes, los coeficientes de las variables uso de Facebook (1.002) y generaciones (0.712) son los más altos, por lo tanto, presentaron la asociación más fuerte con el uso de Apps móviles.

\section{Discusión}

El objetivo central del estudio fue determinar los factores que influyen en el uso de las Apps durante el primer año de la COVID-19. Este estudio utilizó 11 factores, coherentes con la literatura revisada, para desarrollar un modelo de respuesta ordinal. En general, los hallazgos, en la mayoría de casos, fueron los esperados, al mostrarse consistentes con la teoría y evidencia empírica. Las variables uso de Facebook y generaciones están cercanamente relacionadas con la información global que se tiene sobre éstas, al contar con un mayor número de usuarios de redes sociales en el mundo y las tendencias de las generaciones más jóvenes a ocupar un promedio de cuatro horas diarias en su teléfono móvil, respectivamente. Contrariamente, TikTok, la red social que mayor número de descargas presentó a nivel mundial en el 2020, no resultó ser un determinante en el uso de Apps en esta investigación.

Respecto a la variable número de Apps descargadas, el resultado obtenido en este estudio concuerda con el reporte del Instituto Vodafone para la Sociedad y las Comunicaciones (2013), al señalar que desde el lanzamiento de las aplicaciones por parte de los principales proveedores en este mercado las Apps han tenido una gran aceptación entre los usuarios de telefonía celular (App Store y Play Store). En forma similar, se vincula con el informe de We Are Social Inc \& Hootsuite (2021), que menciona que las descargas de aplicaciones móviles aumentaron en un 7\% a nivel mundial. El nivel de educación, como factor determinante del uso de Apps, refleja que el uso de Apps ha tenido fundamental importancia en el sector educativo, dadas las nuevas modalidades de enseñanza-aprendizaje en línea y a distancia.

En cuanto al ingreso, los hallazgos guardan relación con los de Li et al., (2006), quienes señalan a la restricción presupuestaria como uno de los factores claves del uso de Apps, además del tiempo, a la hora de utilizar las aplicaciones móviles para realizar compras en línea a través de éstas. La variable género, pese a no tener significancia estadística, presentó la misma relación que encontraron Morales et al., (2020) y Pontin et al., (2021), quienes sugieren que las mujeres tienden a utilizar en mayor medida las Apps educativas y de salud con respecto a los hombres.

En este estudio, el uso de redes sociales durante el primer año de pandemia presentó una relación positiva con la probabilidad de uso de Apps móviles, este hallazgo coincide con la información otorgada por We Are Social Inc \& Hootsuite (2021), donde se menciona que las redes sociales más utilizadas en el mundo para la comunicación y para estar al día con eventos y noticias son Facebook, YouTube y WhatsApp.

\section{Conclusiones}

La pandemia COVID-19 ha implicado la reinvención de las formas de trabajo, de aprendizaje y de socialización. Los medios digitales han facilitado el funcionamiento continuo de la sociedad y de la economía pese a las estrictas medidas de seguridad, como la cuarentena y el distanciamiento social, y demás implicaciones de la COVID-19, que han puesto de relieve el papel fundamental de las tecnologías de la información y la comunicación en el desarrollo global y local. 
Utilizando una regresión de respuesta ordinal, se determinó que la generación a la que pertenecen los usuarios de Apps, un mayor número de Apps descargadas en dispositivos móviles durante la pandemia, el nivel de educación y el uso de Facebook y Zoom, son los factores que influyen en el uso de Apps móviles en los usuarios de Loja. Este modelo también permitió estimar y analizar los niveles de significancia como la relación entre las variables dependiente e independientes.

Se encontró nuevos e interesantes resultados que aportan a la evidencia empírica revisada y que pueden fundamentar el diseño e implementación de políticas públicas que motiven el uso óptimo de las Apps y el bienestar de la sociedad, así como el desarrollo de nuevos estudios que amplíen la comprensión científica de estos temas relevantes, en tiempos de pandemia y post-pandemia.

Si bien, el uso de Apps ha facilitado actividades laborales, educacionales y de relaciones sociales, y optimiza el tiempo, principalmente de los consumidores, se debe promover su uso racional, dado que puede tener repercusiones negativas en la salud y en el comportamiento psicosocial, principalmente por la desconexión personal que implica.

\section{Referencias}

Aydın, G. Ö., Kaya, N., \& Turan, N. (2015). The Role of Health Literacy in Access to Online Health Information. Procedia - Social and Behavioral Sciences, 195, 1683-1687. https://doi.org/10.1016/j.sbspro.2015.06.252

Báez, A., \& Yariv Brauner, M. (2018). Policy options regarding tax challenges of the digitalized economy: making a case for withholding taxes. SSRN Electronic Journal. http://www.oecd.org/tax/tax-policy/taxchallenges-digital-economy-request-for-input.pdf,

Bernal Peralta, J., Espinoza Villalobos, L., Leo Rossi, E., Quiñones Baraybar, L., \& Moscoso Zegarra, G. (2018). Factores que influyen en el uso de las aplicaciones móviles en instituciones financieras mediante smartphone y su comparación entre las ciudades de Arica (Chile) y Tacna (Perú). 3C Tecnología: Glosas de Innovación Aplicadas a La Pyme, 7(2), 48-61.

https://www.3ciencias.com/articulos/articulo/factores-que-influyen-en-el-uso-de-las-aplicacionesmoviles-en-instituciones-financieras-mediante-smartphone-y-su-comparacion-entre-las-ciudades-dearica-chile-y-tacna-peru/

BID. (2019). BID avala los Principios para el Desarrollo Digital. https://blogs.iadb.org/conocimientoabierto/es/bid-avala-los-principios-para-el-desarrollo-digital/

Bonvalet, C., \& Ogg, J. (2006). Enquêtes sur l'entraide familiale en Europe: bilan de 9 collectes. https://books.google.com/books?hl=es\&lr=\&id=tvHd33ltNQgC\&oi=fnd\&pg=PA154\&dq=Bonvalet, + C., $+\% 26+$ Ogg,+ J. $+(2006) \&$ ots=s433EZT76I\&sig=1XUwwPQkGIMS-nOaxVfbhUFMH8A

Celleri, M., \& Garay, C. (2021). Aplicaciones móviles para ansiedad: una revisión en Argentina. 13, 17-24.

Chuttur, M. Y. (2009). Overview of the technology acceptance model: Origins, developments and future directions. Working Papers on Information Systems, 9(37), 9-37.

http://adam.co/lab/pdf/test/pdfs/TAMReview.pdf

Criteo. (2020). Comportamiento de los usuarios de Apps en 2020: España. Madrid: Criteo.

Deloitte. (2019). Plateauing at the peak The state of the smartphone.

https://www2.deloitte.com/content/dam/Deloitte/uk/Documents/technology-mediatelecommunications/deloitte-uk-plateauing-at-the-peak-the-state-of-the-smartphone.pdf

Díaz-Sarmiento, C., López-Lambraño, M., \& Roncallo-Lafont, L. (2017). Entendiendo las generaciones: una revisión del concepto, clasificación y características distintivas de los Baby Boomers, X Y Millennials. Clío América, 11(22), 188-204. https://doi.org/10.21676/23897848.2440 
Fallis, A. (2013). Economía digital para el cambio estructural y la igualdad. Journal of Chemical Information and Modeling, 53(9), 1689-1699. http://www.cepal.org/Socinfo.

Fuks, M. and Salazar, E. (2008). Applying models for ordinal logistic regression to the analysis of household electricity consumption classes in Rio de Janeiro, Brazil. Energy Economics, 30(4), 1672-1692. https://doi.org/10.1016/j.eneco.2007.09.006

Horoshko, O. I., Horoshko, A., Bilyuga, S., \& Horoshko, V. (2021). Theoretical and Methodological Bases of the Study of the iImpact of Digital Economy on World Policy in 21 Century. Technological Forecasting and Social Change, 166, 120640. https://doi.org/10.1016/j.techfore.2021.120640

INEC. (2021). Población y Demografía. https://www.ecuadorencifras.gob.ec/censo-de-poblacion-y-vivienda/

Instituto Vodafone para la Sociedad y las Comunicaciones. (2013). Impact of Mobile Technologies on Enterprises: Strategies, Success Factors, Recommendations.

ITU. (2021). ITU Council report 2021. https://www.itu.int/reports/council-june2021/

Kabbiri, R., Dora, M., \& Kumar, V. (2018). Mobile phone adoption in agri-food sector: Are farmers in SubSaharan Africa connected? Technological Forecasting and Social Change. https://www.sciencedirect.com/science/article/pii/S0040162517317894

Kumar, H., \& Kumar Yadav, S. (2015). Investigating Social Network as Complex Network and Dynamics of User Activities. International Journal of Computer Applications, 125(7), 975-8887.

Li, J., Zheng, R., \& Chen, H. (2006). ACM: Digital Library: Communications Of The ACM. Communications of the ACM, 49(4), 76-82. https://dl.acm.org/doi/fullHtml/10.1145/353360.353371\#lead-in

Lombardía, P., Stein, G., DI, J. P.-D. de investigación., \& 2008, U. (2008). Politicas para dirigir a los nuevos profesionales-motivaciones y valores de la generacion Y. Academia.Edu. https://www.academia.edu/download/36278444/DI-0753.pdf

Luarn, P., \& Lin, H. H. (2005). Toward an understanding of the behavioral intention to use mobile banking. Computers in Human Behavior, 21(6), 873-891. https://doi.org/10.1016/J.CHB.2004.03.003

Mesenbourg, T. L. (2001). Measuring the digital economy.

Morales, J. C., Ramírez, N. E., Vargas, S. H., Peñuela, A. J., Morales, J. C., Ramírez, N. E., Vargas, S. H., \& Peñuela, A. J. (2020). Uso de aplicativos móviles en el aula y sus factores determinantes. Formación Universitaria, 13(6), 13-22. https://doi.org/10.4067/S0718-50062020000600013

New Strategist Publication, I. (2010). American Generations: Who They Are and How They Live. Ithaca, N.Y.: New Strategist Publications, Inc.

OFCOM. (2016). Adults’ media use and attitudes. http://www.jmir.org/2017/8/e290/

OMS. (2020). Preguntas y respuestas sobre la enfermedad por coronavirus (COVID-19)| Nodo Argentina Campus Virtual de Salud Pública. https://argentina.campusvirtualsp.org/preguntas-y-respuestas-sobrela-enfermedad-por-coronavirus-covid-19

Pew Research Center. (2019). Where Millennials end and Generation Z begins. https://www.pewresearch.org/fact-tank/2019/01/17/where-millennials-end-and-generation-z-begins/

Pontin, F., Lomax, N., Clarke, G., \& Morris, M. A. (2021). Socio-demographic determinants of physical activity and app usage from smartphone data. Social Science \& Medicine, 284, 114235. https://doi.org/10.1016/J.SOCSCIMED.2021.114235

PWC. (2011). Millennials at work Reshaping the workplace. https://www.pwc.com/m1/en/services/consulting/documents/Millennials-at-work.pdf 
Rahimi, R., Khoundabi, B., \& fathian, A. (2021). Investigating the Effective Factors of Using mHealth Apps for Monitoring COVID-19 Symptoms and Contact Tracing: A Survey among Iranian Citizens.

International Journal of Medical Informatics, 104571.

https://doi.org/10.1016/J.IJMEDINF.2021.104571

Rezaei, R., Safa, L., \& Ganjkhanloo, M. M. (2020). Understanding farmers' ecological conservation behavior regarding the use of integrated pest management- an application of the technology acceptance model. Global Ecology and Conservation, 22, e00941. https://doi.org/10.1016/J.GECCO.2020.E00941

Saucedo Soto, J. M., Hernández Bonilla, A., Amezcua Núñez, B., De la Peña de León, A., \& Ávila Rocha, R. V. (2017). COMPORTAMIENTO QUE PRESENTA LA GENERACION SILENCIOSA RESPECTO A LA MODA. Revista Internacional Administración \& Finanzas, 10(5), 95-103. https://ssrn.com/abstract=3040963www.theIBFR.com

Statista. (2020a). Annual number of mobile app downloads worldwide 2020 | Statista. https://www.statista.com/statistics/271644/worldwide-free-and-paid-mobile-app-store-downloads/

Statista. (2020b). Las tiendas de aplicaciones más grandes del mundo 2020 | Statista. https://www.statista.com/statistics/276623/number-of-Apps-available-in-leading-app-stores/

Statista. (2020c). Smartphone penetration worldwide | Statista. https://www.statista.com/statistics/203734/global-smartphone-penetration-per-capita-since-2005/

Stein, Joel. (2013). The Me Me Me generation. Revista Time. http://time.com/247/Millennials-the-me-me-megeneration/

Schwab, K., 2017. The Fourth Industrial Revolution. Currency. Souitaris, V., 2002. Technological trajectories as moderators of firm-level determinants of innovation. Res. Policy 31, 877-898.

Tutz, G. and Berger, M. (2017). Separating location and dispersion in ordinal regression models. Econometrics and Statistics, 2, 131-148. https://doi.org/10.1016/j.ecosta.2016.10.002

We Are Social Inc, \& Hootsuite. (2021). Digital 2021 - We Are Social. In We are cocial and Hootsuite (p. 8). https://wearesocial.com/digital-2021

Wey Smola, K., \& Sutton, C. D. (2002). Generational differences: Revisiting generational work values for the new millennium. Journal of Organizational Behavior: The International Journal of Industrial, Occupational and Organizational Psychology and Behavior, 23(4), 363-382.

Zhao, H. (2020). Tech's response to COVID-19. 